\title{
ANALYSE CRITIQUE DU SYSTEME FISCAL DE LA RDC : \\ FONDEMENT JURIDIQUE ET APPLICATION DE LA LOI
}

\author{
Par Ngoy Ndjibu ${ }^{1}$ et Mutonwa Kalombe Jean-Marc Pacifique ${ }^{2}$
}

\section{INTRODUCTION}

Parler du système fiscal congolais dans un article, serait un travail immense suite à la complexité de ce système qui comprend plusieurs impôts et taxes, qui sont catégorisés en impôt direct et impôt indirect ${ }^{3}$. Ainsi, pour mieux délimiter le thème, nous allons nous consacrer seulement aux impôts que le pouvoir central a cédés aux provinces dans le cadre de l'autonomie financière (régionalisme politique), plus précisément l'impôt foncier et l'impôt sur les revenus locatifs.

Il est utile de rappeler que la constitution congolaise du 18 février 2006 institue le régionalisme politique, dans les relations entre le pouvoir central et les provinces. La décentralisation administrative est consacrée à l'intérieur des Provinces. Ce qui implique logiquement qu'entre le pouvoir central et les Provinces, on ne parle pas explicitement ou implicitement de la décentralisation fiscale.

Pour se développer et s'épanouir, toute société humaine a besoin des institutions, qui devraient gérer la chose publique et bien organiser le pouvoir. La tâche qui incombe à l'Etat est immense et exige des moyens importants tant matériels, humains que financiers. C'est ainsi que le droit fiscal a été institué pour permettre à l'État de se procurer les moyens nécessaires pour sa subsistance, "les finances publiques étant une science de moyens, par lesquels l'Etat et les collectivités publiques se procurent et utilisent les ressources nécessaires à la couverture des dépenses publiques, par la répartition entre les individus des charges qui en résultent $»^{4}$.

1 Docteur en Droit et Chef de Travaux à la Faculté de Droit de l'Université de Lubumbashi, mail: laurentndjibu@yahoo.fr

2 Assistant à la Faculté de Droit de l'Université de Lubumbashi, mail: jeanmarcmutonwa@yahoo.fr

3 Les impôts directs sont payés et supportés par la même personne. Le redevable, celui qui verse le montant de l'impôt, est alors également le contribuable, c'est-à-dire celui qui supporte effectivement l'impôt. L'administration fiscale peut établir la liste des contribuables dans un rôle. En revanche, avec les impôts indirects, comme la taxe sur la valeur ajoutée (TVA) ou les droits de douane, le redevable est distinct du contribuable. Les impôts indirects sont versés par les entreprises ou les personnes redevables, mais répercutés sur le prix de vente d'un produit; ils sont donc supportés par une autre personne, le contribuable.

4 DUVERGER M, finances publiques, 8ième éd., PUF, Paris, 1975, p. 106. 
A cet égard, il faut noter que «la détérioration de la gestion budgétaire en République Démocratique du Congo observée au cours des années antérieures, a fait sentir l'importance capitale des dépenses publiques et la nécessité pour les pouvoirs publics d'avoir une attention soutenue dans ce domaine $»^{5}$.

De façon générale, les finances publiques portent sur les questions d'argent, et ces dernières constituent l'un des problèmes fondamentaux de tout Etat moderne. Aujourd'hui l'impôt est devenu l'un des moyens d'intervention de l'Etat dans la vie économique et sociale. Tout Etat qui se veut fort et stable doit compter sur l'impôt. L'on comprendra par là que si l'application de la loi fiscale est compromise, la conduite de tout le programme de développement économique national ou provincial devient aléatoire.

En effet, la République Démocratique du Congo s'est engagée sur la voie du régionalisme politique des provinces doublé d'une décentralisation à l'intérieur de celles-ci. Pour ce faire, la Constitution de la RDC a reconnu aux provinces une autonomie de gestion financière susceptible de leur procurer les moyens financiers nécessaires pour la couverture de leurs charges publiques.

Parmi les impôts leurs reconnus ${ }^{6}$, nous avons deux impôts (impôt foncier et impôt sur les revenus locatifs) qui frappent soit la possession des biens immobiliers, soit les revenus générés par leur mise en location dont le recouvrement (application de la loi fiscale) pose toujours problème. Ces impôts sont censés constituer les plus importantes sources de revenus des provinces.

De ce constat amer, il nous est venu l'idée de relever le défi en étudiant l'épineuse question de l'application des lois fiscales en matière d'impôt foncier et impôt sur les revenus locatifs, afin de permettre à la RDC à travers ses provinces, d'améliorer qualitativement et quantitativement le rendement de ces deux impôts.

En somme, cette recherche revêt une importance tant scientifique que sociale, parce que cherchant à solutionner un vieux problème en vue d'améliorer la situation financière des provinces de la RDC.

Le pari de cette dissertation est d'aborder l'impôt comme un révélateur des relations entre l'Etat et les citoyens, afin de chercher comment l'impôt foncier et impôt sur les revenus locatifs peuvent s'immiscer dans la vie quotidienne des individus. Pour ainsi permettre cette immixtion, cette recherche se propose de relever les problèmes de mise en œuvre des

5 BAKANDEJA WA MPUNGU, droit des finances publiques, éd. Noraf, Kinshasa, 1997, p. 33.

6 Il faut retenir que c'est le décret-loi 089 du 10 juillet 1998 portant fixation de la nouvelle nomenclature des taxes autorisées aux entités administratives décentralisées, des recettes administratives d'intérêt commun et des recettes fiscales cédées par l'État aux entités, qui a reconnu la compétence exclusive de certains impôts aux entités administratives décentralisées. Mais, ce décret a été abrogé par l'Ordonnance-loi n $013 / 001$ du 23 février 2014 fixant la nomenclature des impôts, droits, taxes et redevances des provinces et des entités territoriales décentralisées ainsi que leurs modalités de répartition. Ainsi, a son article trois il est disposé que : «Les impôts provinciaux et locaux comprennent notamment : l'impôt sur la superficie des propriétés bâties et non bâties; l'impôt sur les véhicules automoteurs; l'impôt sur les revenus locatifs; l'impôt personnel minimum. 
lois fiscales en matière d'impôt foncier et impôt sur les revenus locatifs, afin d'y apporter des pistes de solution.

Dans les lignes qui suivent, nous allons développer dans un premier temps le fondement juridique de l'impôt foncier et impôt sur les revenus locatifs, dans un deuxième temps le problème de recouvrement de l'impôt foncier et l'impôt sur les revenus locatifs et enfin dans un troisième temps les pistes de solution.

\section{FONDEMENT JURIDIQUE DE L'IMPOT FONCIER ET L'IMPOT SUR LES REVENUS LOCATIFS}

Pour la petite histoire, l'imposition de la terre avait toujours été la base des systèmes fiscaux. En effet, comme il est décrit par WINFREY (1973), ainsi que par BUCHANAN et de FLEURS (1975) cités par DAVID CAMERON ${ }^{7}$, les impôts fonciers étaient l'une des premières formes de taxation à prendre en considération. Les économistes ont conclu que la propriété, et plus particulièrement la terre, était la seule base logique pour la taxation. Les physiocrates voyaient les terres agricoles comme le seul secteur productif. Et aujourd'hui, il est admis que les impôts fonciers constituent la principale ressource financière importante pour les entités décentralisées.

En République Démocratique du Congo, le régime fiscal applicable au domaine foncier et immobilier est essentiellement défini par deux textes de base, à savoir :

- l'ordonnance-loi n 69-006 du 10 février 1969 relative aux impôts réels, telle que modifiée et complétée à ce jour;

- l'ordonnance-loi n 69-009 du 10 février 1969 relative aux impôts cédulaires sur les revenus telle que modifiée et complétée à ce jour.

Ces deux textes ont institué, en matière d'imposition foncière et immobilière, trois types d'impôt qui portent sur :

- la superficie des propriétés foncières bâties et non bâties (impôt foncier);

- la superficie des concessions minières et d'hydrocarbures;

- les revenus provenant de la location des bâtiments et terrains situés sur le territoire congolais (impôt sur les revenus locatifs).

En ce qui nous concerne, nous allons développer seulement l'impôt foncier et l'impôt sur les revenus locatifs faisant l'objet de cette recherche.

\section{IMPOT SUR LES REVENUS LOCATIFS}

Conformément aux dispositions du circulaire ministérielle 0023/CAB/MIN/FIN\&BUD/ 2001 du 9 janvier 2001 relative à l'application du décret- loi 109-2000 du 19 juillet 2000 modifiant et complétant certaines dispositions de l'article 4 de l'ordonnance-loi no 69-009 du 10 Février 1969 relative aux impôts cédulaires sur les revenus, l'impôt sur les revenus

7 CAMERON D., Property taxes, Willamette University, College of Law, 1999, p.95. 
locatifs s'applique non seulement aux revenus de la location et de la sous-location de bâtiments et terrains, mais également au cas de mise à disposition à titre gratuit des bâtiments et terrains en faveur d'une entreprise ou de toute autre personne pour usage professionnel, situés au Congo, quel que soit le pays du domicile ou de la résidence du bénéficiaire.

Par usage professionnel, il faut entendre, au sens du décret-loi susvisé, toute exploitation ou activité poursuivant un but lucratif. Il importe de préciser que toute mise à disposition des bâtiments et terrains au profit d'une entreprise sociétaire ou individuelle, quel qu'en soit l'usage (commercial ou industriel), est réputée usage professionnel et donc imposable à l'impôt sur les revenus locatifs.

Toutefois, il convient de souligner que l'utilisation par une personne physique d'un bâtiment ou terrain lui appartenant, dans le cadre de l'exploitation d'une entreprise individuelle, ne peut être considérée comme un cas de mise à disposition à titre gratuit, imposable à l'impôt sur les revenus locatifs.

Par ailleurs, ne sont pas concernés par l'impôt sur les revenus locatifs les revenus, la location des bâtiments et terrains appartenant aux sociétés immobilières, étant donné qu'ils sont imposés à l'impôt professionnel sur les bénéfices et profits.

Il est dû par le propriétaire, le possesseur ou le titulaire d'un droit réel immobilier ou le bénéficiaire du profit brut de la sous-location des bâtiments et terrains.

Pour l'imposition, il est tenu compte des revenus bruts annuels auxquels est appliqué le taux de $22 \%$.

Le législateur a institué un système de retenue à la source opéré par le locataire sur les loyers versés au bailleur. Le taux de la retenue est de $20 \%$ du montant du loyer et payable dans les dix jours qui suivent le paiement du loyer, le solde soit $2 \%$ étant versé par le bailleur avant le 1er février.

Par ailleurs, il est institué des bases forfaitaires d'imposition qui se substituent aux loyers déclarés lorsque ceux-ci se révèlent inférieurs à ces bases.

Des exemptions à l'impôt sur les revenus locatifs sont prévues en faveur notamment de l'Etat et de ses démembrements, des associations sans but lucratif, des organismes internationaux.

\section{IMPOT FONCIER}

Conformément aux dispositions de l'article premier de l'ordonnance-loi n69-006 du 10 février 1969, relative aux impôts réels telle que modifiée à ce jour, Il est établi un impôt foncier assis sur deux bases ${ }^{8}$ : la superficie bâtie et la superficie non bâtie. Il est annuel et dû par toute personne physique ou morale, titulaire du droit de propriété, de possession, d'em- 
phytéose, de superficie, de cession, de concession ou d'usufruit des biens imposables et situées sur le territoire national ${ }^{9}$.

Il frappe aussi toutes personnes occupant, en vertu d'un bail, des biens immobiliers faisant partie soit du domaine privé de l'Etat, des Provinces, des Villes et des Communes, soit du patrimoine des circonscriptions. Sont pris en compte dans l'imposition, les éléments qui existent au 1er janvier de l'année que les redevables sont tenus de déclarer auprès de l'administration au plus tard le ler février.

Il est annuel et dû par toute personne physique ou morale, titulaire du droit de propriété, de possession, d'emphytéose, de superficie, de cession, de concession ou d'usufruit des biens imposables ainsi que par les personnes occupant, en vertu d'un bail, des biens immobiliers faisant partie soit du domaine privé de l'Etat, des Provinces, des Villes et des Communes, soit du patrimoine des circonscriptions.

Sont pris en compte dans l'imposition, les éléments qui existent au 1er janvier de l'année que les redevables sont tenus de déclarer auprès de l'Administration au plus tard le 1er février.

La législation fiscale congolaise prévoit des exonérations et exemptions en faveur de certaines propriétés soit en raison de la qualité de leurs propriétaires, soit en raison de leur affectation. Ainsi à titre d'illustration, les propriétés appartenant à l'Etat et à ses démembrements, aux Etats étrangers, aux associations sans but lucratif ainsi que les immeubles affectés exclusivement à l'agriculture ou à l'élevage sont exonérés de l'impôt foncier.

L'imposition des propriétés foncières a subi une évolution à travers le temps. En effet, jusqu'au la mise sur pied de la DRKAT au Katanga, les propriétés foncières sont imposées suivant deux modes de calcul ci-après : le système d'imposition par superficie au mètre carré et le système d'imposition forfaitaire.

Le système d'imposition par superficie au mètre carré concerne uniquement les villas et consiste à les imposer en fonction de la superficie bâtie par application des taux fixés au mètre carré suivant le rang des localités. L'impôt foncier sur la superficie des villas est calculé aux taux ci-après par mètre carré de superficie:

- dans les localités dites de premier rang: 1,50 UDS/m2;

- dans les localités dites de deuxième rang: $1 \mathrm{USD} / \mathrm{m} 2$;

- dans les localités dites de troisième rang: 0,50 USD/m2;

- dans les localités dites de quatrième rang : $0,30 \mathrm{USD} / \mathrm{m} 2$.

Par contre, le système d'imposition forfaitaire vise toutes les autres propriétés bâties et non bâties (immeubles et terrains) à l'exclusion, bien entendu, des villas.

Il consiste à appliquer à ces propriétés des taux forfaitaires dont le montant variait suivant le rang des localités. Le taux est de :

9 Ceci s'explique par le fait que le système fiscal congolais applique le principe de la territorialité d'impôt, selon lequel la matière imposable concernant un impôt doit se trouver su le territoire de la $\mathrm{RDC}$, quelle que soit la nationalité du contribuable au bas de page commentaire. 
- dans les localités dites de premier rang: 75 USD;

- dans les localités dites de deuxième rang : 37,5 USD;

- dans les localités dites de troisième rang: 18,75 USD;

- dans les localités dites de quatrième rang : 9,35 USD.

Pour des propriétés non bâties, le taux est de :

- 30 USD pour les terrains situés dans les localités de 1er rang;

- 4,50 USD pour les terrains situés dans les localités de 2e rang;

- 2 USD pour les terrains situés dans les localités de 3 e rang;

- 1,50 Ff pour les terrains situés dans les localités de 4e rang.

\section{LE PROBLEME DE RECOUVREMENT DE L'IMPOT FONCIER ET IMPOT SUR LES REVENUS LOCATIFS}

Considérée il y a quelques années comme un coût à subir passivement, la fiscalité est devenue un paramètre nécessaire à la gestion de toute organisation, paramètre dont l'utilisation judicieuse procure une richesse certaine, du fait essentiellement des incidences qu'il peut avoir en termes de flux de trésorerie.

La fiscalité se concrétise donc par le paiement de l'impôt sur le résultat dégagé de l'activité et elle constitue pour la société une charge à gérer tout en optimisant les différents choix fiscaux. C'est par le jeu de différents choix entre des techniques fiscales ou entre des techniques juridiques préalables, que les objectifs de neutralisation et de régularisation de la charge fiscale peuvent être atteints.

Après un siècle de colonisation, près de cinquante ans d'indépendance, les Etats africains en général, la RDC en particulier, semblent être dans une incapacité à gérer un système fiscal digne de ce nom. Certes, quelques efforts sont perceptibles ici et là. Mais, la crise de crédibilité du droit fiscal demeure le dénominateur commun.

Depuis leurs conceptions, l'impôt sur les revenus locatifs et l'impôt foncier ont connu plusieurs problèmes en ce qui concerne le recouvrement.

Pour ce qui est de l'impôt sur les revenus locatifs, dans un premier temps, on a réduit le taux de l'impôt de 30 à $22 \%$ en enlevant toutes les charges locatives, dans un deuxième temps, généralisé le système de la retenue à la source qui, en son temps, ne concernait que les contribuables qui avaient des locataires personnes morales et enfin dans un troisième temps, la cession exclusive aux provinces (Entités Administratives Décentralisées) ${ }^{10}$.

10 La décentralisation administrative vise à refaire la répartition, à différents échelons de gouvernement, de responsabilités et de ressources financières, pour assurer la fourniture de services publics. C'est le transfert de responsabilité de la planification, du financement et de la gestion liée à certaines fonctions du gouvernement central et de ses organes vers des unités d'administration sur le terrain, des cellules ou niveaux subalternes de l'administration, des autorités publiques semi-autonomes, des municipalités ou des régions. Les trois principales formes de décentralisation administrative : la déconcentration, la délégation et la dévolution. 
En ce qui concerne l'impôt foncier, sa législation n'a pas trop connue des réformes comme c'est le cas de l'impôt sur les revenus locatifs. Il est à note que malgré le souci du législateur d'appliquer un taux forfaitaire, recouvrement pose toujours problème à cause de la présence manifeste des maisons de fortune ainsi qu'à cause de la présence d'une économie de subsistance que connaisse la RDC.

Néanmoins, nous pouvons dire que dans l'ensemble, le fondement juridique de l'impôt foncier et l'impôt sur les revenus locatifs n'est pas aussi mauvaise. Mais, si l'élargissement de l'assiette fiscale et le recouvrement posent problème et font que les recettes sont faible, c'est à cause notamment de :

\section{L'ABSENCE DU CADASTRE FISCAL}

Le cadastre, est certes une institution trop citée et trop peu connue. Elle est entourée d'incompréhension, de préjugés, qui vont, quelquefois, jusqu'à un discrédit ${ }^{11}$. Il joue trois rôles majeurs, à savoir : fiscal; civil et social. Le cadastre fiscal est un système d'information foncière qui permet d'assurer la gestion des données et informations nécessaires à l'évaluation des biens fonciers, en vue de la détermination de la taxe foncière ou afférente au foncier. L'information requise porte sur :

a) les éléments permettant d'apprécier la valeur (locative ou vénale) de la propriété, et;

b) l'identification du contribuable (le propriétaire du bien ou l'occupant).

L'inventaire du cadastre fiscal ne doit donc pas seulement être descriptif, exhaustif et permanent; il doit être aussi évaluatif ${ }^{12}$. En somme, la ville de Lubumbashi n'a pas de cadastre fiscal, le cadastre est jusque là utilisé dans son aspect civil. Il est donc nécessaire, d'y penser si on veut bien maximiser les recettes fiscales provenant de la fiscalité immobilière. Mais il faut retenir que, l'ordonnance-Loi n 69-006 du 10 février 1969 sur l'impôt réel telle que modifiée et complétée à ce jour tente dans une certaine mesure à l'instauration d'un cadastre fiscal atypique. Ainsi, pour recouvrer l'impôt foncier, l'ordonnance précitée a institué un impôt forfaitaire annuel, dont le montant varie suivant la nature des immeubles et le rang des localités. A ce titre la RDC sur le plan fiscal est subdivisée en 4 rangs de localité.

\section{L'ADMINISTRATION FISCALE (DRKAT ${ }^{13}$ )}

Comme l'affirme G. Ardant, « on a trop souvent tendance à oublier que le système fiscal le mieux conçu ne vaut que par l'administration qui le met en ouvre ${ }^{14}$. Dans les pays

11 MARSTBOOM L., Le cadastre et l'impôt, Wester, Bruxelles, 1946, p.4.

12 LASSERVE D., Conditions de mise en place des systèmes d'information foncière dans les villes d'Afrique sub-saharienne francophone, publié pour le Programme de gestion urbaine par la Banque mondiale, Washington, D.C., mars 1994, pp. 12-13.

13 Direction des recettes du Katanga.

14 ARDANT G., Théorie sociologique de l'impôt, S.E.V.P.E.N, Paris, 1965, p.849. 
d'Afrique, si l'emprise des administrations fiscales sur la mise en œuvre de la fiscalité demeure un fait indéniable, il est aussi communément admis que ces administrations « fonctionnent dans des conditions insatisfaisantes et ne sont pas plus performantes aujourd'hui qu'elles ne l'étaient dans la période postérieure aux indépendances (années soixante) » ${ }^{15}$. En effet, sous l'effet conjugué de la " sous-administration $»^{16}$ et de la « mal administration $»^{17}$ fiscale, aggravé par une imitation de façade, dans ces pays, « l'administration fiscale déforme et rend difforme la fiscalité.

Or, partant de cette conception, la situation au Katanga se présente de la manière suivante : un faible personnel peu qualifié et des services inorganisés sont chargés d'appliquer des textes inadaptés. En effet, Il faut observer que la Direction des Recettes du Katanga, jusqu'à ce jour reste figurante et son effectivité par rapport au recouvrement de l'impôt foncier et l'impôt sur les revenus locatifs pose problème. Elle connait les mêmes problèmes que ceux qu'a connus l'ancien service de mobilisation des recettes créé en 2004. Ces problèmes sont répertoriés dans l'exposé de motifs de l'édit n 0004 du 25 septembre 2009 portant création de la direction des recettes du Katanga :

- Inexistence des fichiers ou répertoire des assujettis au paiement des impôts provinciaux;

- Non maîtrise, voire absence des données statistiques afférentes à plusieurs taxes;

- Insuffisance des effectifs par rapport à l'abondance de la matière et à l'étendue de la province;

- Manque de moyens logistiques nécessaires;

- Insuffisance de professionnalisme en matière fiscale des agents commis à la tâche.

Les Services de cette Direction des Recettes sont encore concentrés dans les agglomérations urbaines où sont situées les matières imposables à l'impôt foncier et à l'impôt sur les revenus (immobiliers).

15 RAMBOURG M., «L'échec des réformes en Afrique. Le cas des pays francophones au sud du Sahara », Revue Française d'Administration Publique, juillet-septembre 1998, p. 405.

16 ANGE BANGO, L'élaboration et la mise en œuvre de la fiscalité dans les pays de la communauté économique et monétaire de l'Afrique centrale (CEMAC), Thèse, Université Jean Moulin Lyon 3, 2009, p.174 : Il existe quatre cas de figure de la sous-administration qui n'existent jamais évidement à l'état pur dans la pratique administrative des pays en développement : le premier qui est le plus fréquent « se résume en la combinaison d'une forte différentiation des structures administratives et d'une faible intégration sociale ». Le deuxième est inverse au précédent et se résume dans la formule suivante : forte intégration, plus faible différentiation. Le troisième « correspond lui aussi à une crise dans la répartition des ressources entre les citoyens mais la crise est due, cette fois non à l'insuffisant équipement administratif, mais à l'immensité des attentes dont l'appareil administratif est l'objet (...) ». Enfin, le dernier cas de figure « s'analyse en l'existence d'un appareil administratif limité à l'exercice de sa fonction de domination et ressenti comme étranger par ceux à l'égard desquels il exerce son autorité». Tous ces cas de figure peuvent s'appliquer au Katanga.

17 NGAOSYVATHN P., Le rôle de l'impôt dans les pays en voie de développement, Tome1 LGDJ, 1974, pp. 146-183. Tout en soulignant que le mot mal-administration n'est pas employé souvent directement et semble usité beaucoup plus par référence au concept de « mauvaise administration». 


\section{DES FACTEURS ECONOMIQUES}

L'institution d'un système fiscal de rendement élevé n'est possible que dans une économie comportant un certain degré de développement industriel. L'observation montre que le développement d'un système fiscal moderne constitué, dans des proportions variables, par l'impôt sur le revenu et l'impôt sur la dépense n'est donc possible qu'avec l'essor industriel $^{18}$.

Il se dégage de ce propos que le niveau de développement influe largement sur le rendement et la structure d'un système fiscal. De ce fait, lorsqu'un pays, en l'occurrence la RDC, n'a pas le niveau de développement voulu, le rendement fiscal devient hypothétique.

La conjoncture exerce une influence sur l'incidence de l'impôt en général et en particulier l'impôt foncier et l'impôt sur les revenus locatifs, dont les conséquences se font sentir sur les recettes fiscales. "Un impôt sera dit sensible à la conjoncture lorsque son rendement varie en fonction de la situation économique et notamment des variations cycliques de celle-ci. Cette sensibilité dépend évidemment des données économiques constituant la matière imposable, qui sont plus ou moins sensibles à la conjoncture $»^{19}$.

En effet, lorsque nous nous basons sur les résultats d'observation en général et sur les entretiens informels réalisés avec les agents de la Direction de Recettes du Katanga, il se dégage que l'impôt sur les revenus locatifs et l'impôt foncier ne sont pas recouvrés conformément à l'esprit de la loi fiscale, entre autre, à cause du niveau de vie des contribuables (économie de subsistance). Par conséquent, l'administration fiscale rend ces deux impôts sélectifs.

Ce qui pousse M. NYABIRUNGU MWENE SONGA de considérer l'économie congolaise de " criminalisation ». "La criminalisation de l'économie congolaise signifie que celle-ci ne fonctionne plus selon les normes contenues dans nos lois, et que c'est la violation de ces lois qui est devenue la norme régulatrice de la production, de la circulation, de l'échange, de la répartition et de la consommation des richesses $»^{20}$. M. BOSEKOTA W'ATSHIA renchérit en soutenant que : «depuis plusieurs années, l'activité économique en R.D.C est en régression continue. Il s'agit d'une très profonde crise structurelle et non d'un simple phénomène conjoncturel $»^{21}$. A ce titre, il faut observer que cette criminalisation tire aussi ses origines dans l'économie de subsistance que connait la RDC, qui gène excessivement le rendement du système fiscal congolais en général.

Mais, il faut observer que, si le système fiscal dépend, dans une large mesure, des structures économiques globales, il ne faut pas croire qu'on se trouve devant un déterminisme

18 BELTRAME P. et MEHL L., techniques, politiques, et institutions fiscales comparées, 2éd. PUF, Paris, 1997 p.324.

19 BELTRAME P. et MEHL L., op.cit, p. 352.

20 NYABIRUNGU M. SONGA, La criminalisation de l'économie Zaïroise, éd. DES, Kinshasa, 1996, p. 8.

21 BOSEKOTA W'ATSHIA, Rebâtir le Congo Démocratique : De la Bonne Gouvernance Etatique et du Rôle Clé des PME-PMI!, éd. Presses Universitaires « BEL CAMPUS », Tome I, 2004, p. 17. 
sur lequel l'action du législateur n'aurait pas de prise. L'impôt peut donc « non seulement dans une certaine mesure jouer un rôle dans la transformation des structures économiques, mais en outre, les pouvoirs publics sont obligés de rechercher l'amélioration du système fiscal au regard du rendement et de la justice fiscale quel que soit l'état de l'économie $»^{22}$.

En définitive, dans les pays sous développés, les pouvoirs publics sont prisonniers d'une redoutable contradiction : d'une part impossibilité de taxer une population qui manque du nécessaire pour sa propre survie et de l'autre la crainte d'imposer les classes possédantes considérées, à tort ou à raison, comme les seuls gisements de l'épargne nationale ${ }^{23}$. Par conséquent, impossible d'asseoir un impôt sur le revenu à cause de la faiblesse du niveau de vie, le revenu étant largement en dessous du seuil du minimum vital.

\section{DES FACTEURS POLITIQUES}

Les facteurs sociopolitiques jouent négativement sur la pression fiscale. Qu'il s'agisse d'introduire une nouvelle imposition foncière, ou d'apporter des améliorations substantielles, d'importantes décisions doivent être prises. La conception et la mise en œuvre de l'impôt étant des opérations délicates sur le plan politique, des stratégies sont nécessaires.

L'étude de l'environnement sociopolitique de l'impôt doit être divisée en deux volets d'importance inégale. En effet, «la souveraineté fiscale d'un pays en voie de développement est circonscrite par des exigences intérieures et extérieures. Ces contraintes se transforment en des blocages qui dépriment leur fiscalité $\gg 24$.

Techniquement, l'augmentation des recettes requiert la hausse des taux de l'impôt, soit la création de nouveaux impôts ou la modification des anciens impôts; l'amélioration du recouvrement et le renforcement des contrôles. Il a été observé que dans les pays en voie de développement, y compris la RDC, cette hausse n'apparait pas comme une manipulation des techniques fiscales, mais comme un art politique, ce qui explique l'absence d'une philosophie fiscale claire dans ces pays.

Comme le soutient M. NGAOSYVATHN que : «dans les circonstances du sous-développement, le prélèvement fiscal est éminemment un acte politique, un art du possible, c'est-à-dire, un art de pouvoir. Il s'agit de contraindre et de se faire obéir, tant des riches qui défendent leur superflu que les pauvres qui sauvegardent leur nécessaire. Devant cette convergence de la résistance de groupes sociaux aussi opposés, les pouvoirs publics doivent disposer d'une bonne condition politique, pour pouvoir désamorcer les mécontentements et imposer des sacrifices $»^{25}$.

Cet art s'explique par une volonté politique émanant des pouvoirs publics de lever autant d'impôt que l'exigera la réalisation des besoins gouvernementaux. Alors que le prélè-

22 BELTRAME P. et MEHL L., op.cit., p.332.

23 GEFFROY JB., grands problèmes fiscaux contemporains, Puf, paris, 1993, p. 164. .

24 NGOASYVATHN P., op.ict., p. 185.

25 NGOASYVATHN P., op.cit., p.191. 
vement fiscal ne doit pas être qu'un instrument au service d'une fin, puisque dépendant aussi de la philosophie qui l'accompagne. Nous savons que la philosophie unique justifiant un système fiscal est la mobilisation des fonds à mettre à la disposition des dirigeants pour la gestion du pays et garantir l'intérêt général du peuple. La question majeure reste en ce qui concerne la RDC, est celle de savoir si les dirigeants comptent ou pas sur les fonds d'origine fiscale et à quel degré.

De tout ce qui précède, le développement ou le sous développement fiscal d'un pays, n'est qu'un reflet de la force ou de la faiblesse de la volonté politique fiscale. Par conséquence, les techniques fiscales sans volonté politique (philosophie fiscale) de s'en servir sont vaines et la volonté politique sans techniques est destructrice.

\section{DES PROBLEMES D’URBANISATION}

Qu'on l'accepte ou qu'on la rejette, le processus d'urbanisation est déjà bien présent et il doit se poursuivre puisqu'on ne peut pas parler de l'impôt foncier et l'impôt sur les revenus locatifs sans parler des villes. "Ville diluée, ville informe, mal structurée au premier abord, avec d'immenses quartiers étalés sans fin dans le même maillage de rues en damier, une énorme périphérie sous-équipée, une périphérie qui a grandi trop vite autour d'un noyau ancien hérité de la période coloniale, voila l'image que l'on garde le plus souvent des villes de la $R D C »^{26}$.

Sans avoir peur d'être contredit, la ville de Lubumbashi est caractérisée par la coexistence de deux types d'urbanisation : la ville planifiée d'une part, constituée des constructions qui étaient à l'origine réservées aux colons, ainsi que de nouvelles constructions résidentielles qui ont été aménagés après l'indépendance, de l'autre part, la ville spontanée, constituée des constructions nouvelles qui se développent dans les zones non aménagées, sans infrastructures et équipement.

L'urbanisation est manifestement un fait marquant de l'Afrique contemporaine. Elle entraine avec elle des transformations radicales et rapides des structures sociales et économiques ${ }^{27}$. Le fondement pratique de l'impôt foncier et impôt sur les revenus locatifs reste donc le cadastre dans son rôle fiscal, qui aussi dépend du degré d'urbanisation et de la politique d'habitat. De ce fait, si la RDC veut compter sur l'imposition immobilière, il faut nécessairement repenser à la réorganisation totale du service d'urbanisme et d'habitat.

En effet, la construction ou les travaux qu'on envisage doivent être conformes aux règles contenues dans le plan local d'urbanisme de la ville ou à défaut, prévues par le règlement national d'urbanisme.

Il est impossible selon la réglementation, de construire une maison sans permis de construire, sauf peut-être lorsque les travaux sont très discutables, comme par exemple construire une «maison » annexe de $20 \mathrm{~m}^{2}$ en fond de jardin. Malgré la présence de la régle-

26 PAIN M., Kinshasa : la ville et la cité, ORSTOM, Paris, 1984, p.5.

27 GAPYISI E., Le défi urbain en Afrique, Harmattan, Paris, 1989, p.31. 
mentation sur l'autorisation de bâtir, les constructions en RDC en général et Lubumbashi en particulier, s'étalent d'une façon spectaculaire.

Toutefois, malgré cette énorme croissance de la ville de Lubumbashi, soulignons l'absence d'un plan d'urbanisation gouvernemental, surtout dans la commune périphérique. La commune Annexe qui forme la ceinture de la ville, illustre bien le cas de la commune créée sans planification, ni par les topographes de l'administration de la ville, ni par les détenteurs des pouvoirs publics.

\section{LA CORRUPTION DES AGENTS DU FISC}

«Tolérée, encouragée par le système socio-culturel du tiers monde, la corruption entraîne des effets nocifs sur le fisc et la fiscalité. Légitimée et amplifiée par l'environnement des pays en voie de développement, la corruption, dont celle du fisc, résiste aux mesures correctives. Mais, il semble qu'elle pourrait être résorbée par des remèdes drastiques, appliquées avec énergie et constance $»^{28}$.

Le phénomène de corruption en RDC a atteint un niveau systémique qui mérite non seulement une identification secteur par secteur ou acteur par acteur, mais aussi une recherche approfondie des sources de cette dérive ainsi que des motifs de sa reproduction intensive, accélérée et généralisée pendant la transition embrassant toute la vie nationale au niveau de l'Etat, de la société et des individus ${ }^{29}$. Du point de vue historique, le phénomène de la corruption est intimement lié à un déficit de maturité politique hérité de la mauvaise et confuse décolonisation sans un fond de culture de responsabilisation politique et d'engagement idéologique pour la gestion du nouvel Etat. De même, il est lié au manque criant d'une élite nationale de gestionnaires économiques et financiers autant dans le secteur public que privé pendant toute la période coloniale et pour un temps relativement long après l'accession du pays à l'indépendance.

De part de l'observation, il ressort que la corruption du fisc à Lubumbashi est considérée comme un acte normal, un mode de vie tolérable et qui, bénéficie de la triple impunité : politique, administrative et judiciaire. Politique, puisque les pouvoirs sont impuissants pour assainir l'administration. Cette impuissance est justifiée par le fait que, ceux qui sont chargés d'assainir l'administration se trouvent aussi plongé dans la corruption, et chaque fois, ils reçoivent des pots de vin provenant des agents de l'administration. Administrative, puisque cette impunité politique des agents qui trafiquent leur autorité, se double d'une impunité administrative. Le supérieur hiérarchique se livre à la corruption autant que l'agent subalterne. Judiciaire, puisqu'il n'existe pas de pouvoir judiciaire indépendant, mais des juges subordonnés à l'exécutif et participent aux mêmes activités de la corruption ${ }^{30}$.

28 NGAOSYVATHN P., op.cit., p.174.

29 MUZANG KODI, Corruption et gouvernance en RDC durant la Transition (2003-2006), Institut D'études de Sécurité, Paris, 2008.

30 NGAOSYVATHN P., op.cit., pp. 175-176. 
A ce propos, M. RUBBERS soutient que : "Les hauts responsables du fisc se limitent aux rapports avec les grandes entreprises car ils refusent, pour une raison de prestige, de s'abaisser à traiter avec les petits indépendants. D'autre part, les subalternes de l'administration tiennent compte de la puissance virtuelle des citoyens dans l'arène politique : ils préfèrent se montrer prudents avec les " grands » car ils savent que leur supérieur les sacrifiera sans hésiter en cas de problème $»^{31}$.

Ce qui poussent M. KIBANDA MATUNGILA et Cie à affirmer que : "le monstre corruption, c'est l'image d'une société ou d'un organisme qui au lieu de jouer un rôle de générer une société toujours renouvelée, de construire un développement qui tienne compte de la dignité de l'homme, de la justice distributive, de la moralité, des institutions de promotion des valeurs, écoles, entreprises, gouvernance, institutions civiles de protection sociale et de lutte contre la pauvreté, le fait contrairement à la nécessité d'une vie collective positive, pouvant entraîner un progrès soutenu. La corruption est donc un véritable monstre en $R D$. Congo étant donné qu'elle a dépouillé toute les personnes de leurs capacités de générer un habitat nouveau, des conditions de vie dignes et décentes, de développer l'esprit créatif dans toutes les institutions et de supprimer les vices qui ont fini par ronger l'avenir de tout le pays $»^{32}$.

A cet effet, dans le souci de renforcer le civisme fiscal et de lutter contre la corruption, un protocole d'accord a été signé entre le ministère des finances et le comité permanent des évêques de la conférence épiscopale nationale du Congo CENCO), visant la mise en place d'un cadre de concertation de la collaboration entre les deux partie en vue de la campagne de sensibilisation au civisme fiscal. Parmi les activités de la campagne conformément à l'article premier du protocole, figurent l'information et la formation des citoyennes et citoyens congolais sur la nécessité de payer l'impôt et sur leur implication dans la lutte contre la corruption. La durée de ce protocole est deux ans renouvelables, conformément aux dispositions de l'article 5 .

Mais, il faut remarquer que ce protocole souffre de beaucoup d'insuffisance dans son aspect pratique, ce qui fait que ce protocole n'a pas donné des fruits escomptés depuis sa mise en vigueur le 13 juillet 2010. En effet, l'Etat congolais conformément à sa constitution est un Etat laïc, par conséquent ce protocole dans son application va concerner plus les chrétiens catholiques que les chrétiens d'autres églises et les non chrétiens. Ensuite, l'église ne peut seulement pousser les contribuables au civisme fiscal, en mettant de coté les gouvernants qui doivent faire montre d'un bon usage des recettes fiscales. Enfin, le protocole a été signé pour deux ans renouvelables après évaluations par les deux parties, ce qui n'est pas le cas jusqu'aujourd'hui. En définitive, ce protocole n'a apporté aucun impact sur la lutte contre la corruption, puisque depuis quatre ans la situation demeure la même.

31 RUBBERS, B., « Retour sur le «secteur informel». L'économie du Katanga (Congo-Zaïre) face à la falsification de la loi », Sociologie du travail, vol. 49, n³, Elsevier Masson SAS All, 2007, p.8.

32 KIBANDA MATUNGILA et Cie, Le monstre appelé corruption, l'institut des stratégies pour le développement durable, Kinshasa, 2005, p.69. 


\section{DU CONTRIBUABLE}

La fiscalité a deux sujets. L'administration et le contribuable. L'une est étudiée. L'autre est ignoré. Si bien qu'en étant au centre du phénomène fiscal, le contribuable reste inconnu. Le terme contribuable recouvre un contenu sociologique et juridique très complexe. Ainsi, un épicier de village est contribuable au même titre qu'un supermarché. Ce sont pourtant deux réalités tout aussi différentes ${ }^{33}$.

Il est inconcevable, voire impossible de parler de l'impôt sans parler du contribuable, dans la mesure où c'est justement à lui, qu'incombe la charge de payer l'impôt.

On peut, dire que le contribuable fait partie de l'appareil administratif de l'impôt, puisqu'il est souvent l'acteur de l'établissement de l'impôt. Son intervention se manifeste à tous les stades de l'acte d'imposition, qu'il s'agisse de l'assiette, de la liquidation ou du recouvrement de l'impôt ${ }^{34}$.

L'existence de tout système fiscal a pour finalité l'acquittement correct par le contribuable de ses obligations fiscales. Le contribuable devient alors, l'acteur le plus déterminant dans la mise en œuvre de tout impôt. De ce fait, si ce dernier ne se trouve pas dans les conditions idéales, il se retrouvera dans l'obligation d'utiliser de tout moyen sa disposition pour éviter l'impôt.

Il paye d'une part, et de l'autre part, il doit bénéficier de ses effets. Donc, ce sont les effets (la destination de son argent) qui au fait, motivent ce dernier de s'acquitter sans remords. C'est à ce titre que M.GONIDEC cité par M. FOSTING, estime que « la perception fiscale doit aussi se rapprocher que possible de l'utilisation qui sera faite des fonds ainsi collectés ». C'est ainsi que l'adéquation entre ponction fiscale et réalisations publiques se présente comme facteur mobilisateur et légitimant de l'impôt dans les nouveaux Etats, à condition que les pouvoirs publics déchiffrent le message qui remonte ainsi du peuple ${ }^{35}$.

Le rapport du contribuable au système fiscal est d'abord un problème de perception que le citoyen se fait de l'ensemble des prélèvements qu'il doit acquitter. Cette représentation reste essentiellement caractérisée par un sentiment de contrainte, voire d'oppression, qui explique la permanence du phénomène de rejet dont l'impôt est l'objet ${ }^{36}$.

\section{PISTE DE SOLUTION}

Nous n'aurons pas à donner les solutions à tous les problèmes exposés dans la section précédente. D'emblée, les actes contraires aux problèmes soulevés, constitueront des solutions d'une manière générale. L'évaluation du système fiscal devrait faire ressortir les insuffisances en vue de les dépasser et d'insister sur ses aspects positifs pour les consolider; la

33 MARTINEZ JC. et DI MALTA P., Droit fiscal contemporain : l'impôt, le fisc, le contribuable, T1, Litec, Paris, 1986, p.326.

34 BELTRAME P. et MEHL L., op.cit., p. 570.

35 FOSTING JB., FOSTING JB., Le pouvoir fiscal en Afrique, LGDJ, Paris, 1995, p.292.

36 GEFFROY JB., op.cit., p.548. 
finalité étant d'élargir la base et de rehausser le niveau d'équité fiscale en matière d'impôt foncier et impôt sur les revenus locatifs.

Il existe un lien étroit entre la fiscalité et les éléments économiques sur lesquels elle repose qui constituent la matière imposable et que leur nature et leur importance fixent impérativement les formes et les limites des prélèvements possibles.

De ce fait, la structure du système fiscal outre son aspect législatif, dépend fréquemment de la situation économique dans laquelle il doit s'appliquer. «Le niveau de développement économique, la diversité des modes de production de la richesse influent nécessairement sur la répartition du prélèvement fiscal, sur son évolution technique et bien évidement sur son rendement. Ainsi le développement des impôts coüncidait avec le passage de l'économie agricole à l'économie industrielle, de l'économie en nature à l'économie monétaire $»^{37}$.

Il est donc clair, qu'un système fiscal est tributaire de la situation économique dans lequel il est censé se réaliser. C'est à ce titre, que Beltrame en classifiant les systèmes fiscaux sous le critère socio-économique en trois catégories : systèmes fiscaux des pays industriels, systèmes fiscaux des PVD, systèmes fiscaux des pays en économie en transition, soutient que les systèmes fiscaux des PVD, ont été calqué sur le modèle des colonisateurs sans tenir compte des conditions socio-économiques. Or, les prélèvements obligatoires qui composent ces systèmes, ne peuvent être appliqués dans une économie de subsistance, comme c'est le cas de la RDC.

Il faut une réforme fiscale qui tiendra compte de l'aspect socio-économique de la RDC pout créer et établir les prélèvements fiscaux. Ainsi, pour une bonne maximisation comme le propose M. MPOY, l'impôt sur les revenus locatifs peut être incorporé dans l'impôt foncier, quitte à faire une distinction entre la valeur cadastrale pour l'impôt foncier et la valeur locative pour l'impôt sur les revenus locatifs. Mais, en ce qui concerne l'impôt sur les revenus locatifs, il faut associer le ministère de l'habitat pour la gestion efficace de l'impôt sur les revenus locatifs. En effet, du fait qu'il dresse les fiches de locataire, ce ministère peut aider l'administration fiscale à recouvrer optimalement cet impôt ${ }^{38}$. Pour l'impôt foncier, le gouvernement central doit évaluer le potentiel fiscal par rapport à la superficie du pays ${ }^{39}$.

Mais, il faut observer que seule la reforme du fondement juridique ne suffise, il faut en autre penser aux problèmes de mise en œuvre qui gênent en soit une bonne application de la loi fiscale. Il faut donc :

1. L'administration fiscale doit exercer en son sein des activités foncières (évaluation des valeurs des bâtiments et terrains), unique solution pour élargir considérablement l'assiette de l'impôt foncier et l'impôt sur les revenus locatifs. En effet, les services du ca-

37 Idem, p.163.

38 MPOY KADIMA, La rationalisation du système fiscal et douanier comme mécanisme relanceur de l'économie. Principes et outils d'une politique fiscale et douanière développementaliste, Editions Universitaires Africaines Kinshasa, Kinshasa, 2012, p. 229.

39 MPOY KADIMA, op.cit., p. 226. 
dastre et de la conservation ont pour mission de recenser les terrains et bâtiments, d'en préciser la propriété, leur destination ainsi que leur valeur qui permettent de déterminer les valeurs locatives et cadastrales. Ces valeurs locatives ne sont l'œuvre ni du cadastre, ni de la conservation, et ni moins de l'urbanisme. Par contre, l'œuvre de l'administration fiscale, dans son rôle de cadastre, c'est-à-dire la capacité de manipuler avec précision les matrices cadastrales.

2. Politique d'habitat effective et réaliste. L'absence de cette politique d'habitat est un véritable handicap pour le recouvrement de l'impôt, puisqu'on ne sait pas réaliser un recensement de tous les redevables. Grace à cette politique, on devrait être capable de connaître dans un quartier donné, quelles sont, par exemple les maisons qui sont destinées à la location. L'observation de la ville de Lubumbashi montre avec force que cette situation ne peut durer, que l'enjeu fondamental de cette ville est la maîtrise de son développement, de ses constructions, de son habitat. Une culture de la gouvernance urbaine doit rapidement évoluer, elle doit se doter des moyens intellectuels, techniques, légaux et réglementaires afin de maîtriser les possibles.

3. Une politique qui joue positivement sur la pression fiscale. Malgré les difficultés économiques, la politique prime sur les autres aspects de la vie, ce qui rend difficile une quelconque modification. A ce sujet, SREINMO, cité par Bernard SALANIE, affirme que «les systèmes politiques conditionnent la politique fiscale des Etats ${ }^{40}$. Pour ce faire, il faut un aménagement de l'imposition afin que la perception fiscale soit aussi rapprochée que possible de l'utilisation qui sera faite des fonds ainsi collectés.

4. Une adéquation culturelle du système fiscal à l'environnement qui est rarement considérée comme condition de sa bonne application, alors que le phénomène fiscal est aussi culturel qui implique une relation entre le pouvoir et la population. De ce fait, une culture fiscale est de mise, puisque « toute société tend en effet à définir un système de valeurs, d'objectifs et d'impératifs qui caractérisent son idéal politique. Cette axiologie comporte des applications au domaine de l'impôt qui se traduit par les notions de devoir fiscal et de justice fiscale. La première valeur se rapporte aux obligations $d u$ contribuable et tend à légitimer la contrainte qui s'exerce sur lui; la seconde fonde les droits du contribuable et les garanties qui lui sont dues en face du pouvoir $»^{41}$.

5. Les facteurs psychologiques qui prennent en compte la psychologie du contribuable qui tient à ce que l'on a appelé parfois « la mentalité fiscale des peuples ». Elle dépend, plus directement du milieu socio-professionnel du contribuable, du niveau auquel il se situe dans ce milieu et des conditions dans lesquelles il est assujetti à l'impôt ${ }^{42}$. La réduction des tensions psychologiques entre le contribuable et le fisc demande sans doute que celui-ci accorde une plus grande attention aux relations humaines. Cette réduction des tensions requiert davantage une éducation du citoyen qui devra prendre conscience

40 SALANIE B., théorie économique de la fiscalité, Economica, Paris, 2002, p.7.

41 BELTRAME P. et MEHL L., op.cit., p.629.

42 Idem., p. 660. 
de l'importance de l'impôt dans un régime qui reste libéral, tout en sauvegardant l'autorité et la mission de l'Etat ${ }^{43}$.

6. Une collaboration franche entre le fisc et le contribuable est de mise. Cette collaboration se justifie par le fait que, forte du caractère régalien de sa mission, l'administration fiscale a traditionnellement entretenu avec des contribuables des relations fortement teintés d'unilatéralisme. La réduction des tensions psychologiques entre le contribuable et le fisc demande sans doute que celui-ci accorde une plus grande attention aux relations humaines. Cette réduction des tensions requiert davantage une éducation du citoyen qui devra prendre conscience de l'importance de l'impôt dans un régime qui reste libéral, tout en sauvegardant l'autorité et la mission de l'Etat ${ }^{44}$.

7. en ce qui concerne l'administration fiscale, la réussite de la réforme fiscale suppose d'abord une élimination ou du moins une atténuation des obstacles inhérents à l'administration fiscale, dont la plus déterminante est la corruption. La modernisation de l'administration apparaît comme une nécessité impérieuse. A cet égard, le culte de la compétence et de l'intégrité doit être au centre de l'action des pouvoirs publics. Une telle politique, pour produire les résultats escomptés, nécessite une rénovation des autres moyens d'action du fisc ${ }^{45}$. Parmi les autres moyens d'action des administrations fiscales, qu'il convient de rénover, nous avons non seulement des moyens juridiques et techniques, mais également des moyens structurels et matériels. Si la mise en œuvre de l'impôt comporte des actes de puissance publique, créant des obligations unilatérales à la charge du contribuable, des garanties doivent être accordées au contribuable pour le protéger de l'arbitraire. Ces garanties découlent de la notion de légalité qui domine les rapports entre l'Etat et les citoyens dans les nations modernes ${ }^{46}$.

\section{CONCLUSION}

Dans ce présent article, nous nous sommes proposés d'étudier le fondement juridique l'impôt sur les revenus locatifs et l'impôt foncier afin de relever les problèmes de mise en application des lois fiscales en la matière pour aboutir à une bonne effectivité desdites lois.

Comme dans la plupart des pays africains de tradition administrative française ou belge, la fiscalité foncière des provinces de la RDC est encore largement fondée sur les impôts fonciers des propriétés bâties et non bâties et sur les revenus locatifs. Le régime fiscal de ces impôts présente l'inconvénient d'une grande complexité, qui a rendu leur application difficile. De plus, en RDC les Administrations financières ne disposent pas des moyens pour appliquer des fiscalités de masse complexe, dont d'ailleurs le potentiel de recettes est faible relativement aux coûts administratifs de collecte.

43 Idem, p. 667.

44 BELTRAME P.et MEHL L., op.cit., p. 667.

45 ANGE BANGO, op.cit., p.370.

46 BELTRAME P.et MEHL L., op.cit., p. 582. 
Contrairement aux pays développés, où le rôle relatif de la fiscalité foncière dans le financement des collectivités locales tend à décliner, la fiscalité foncière urbaine devrait constituer une recette essentielle pour financer les collectivités locales de la RDC.

Ainsi, malgré ces difficultés, la RDC a l'obligation de repenser la manière d'imposer l'impôt foncier et l'impôt sur les revenus locatifs ainsi que les mécanismes de mise en œuvre.

Partant du postulat selon lequel, un système fiscal est tributaire du niveau de vie socioéconomique, il sera donc, de bonne politique aussi pour nous d'adapter notre système fiscal aux conditions socio-économiques qui nous sont propres. Cette adaptation nous conduira au respect de l'une des caractéristiques d'imposition des revenus dans les pays en voie de développement, celle de la simplification des règles d'assiette fiscale comportant notamment l'institution d'impôts forfaitaires ${ }^{47}$. Cette simplification de l'assiette fiscale, tentera de ne pas porter atteinte au minimum vitae des contribuables et constituera pour l'administration fiscale une compensation à l'évaluation fiscale rigoureuse sur les revenus généralement trop bas.

Pour ainsi simplifier les règles d'assiette fiscale en ce qui concerne l'impôt sur les revenus locatifs et l'impôt foncier, qui du reste frappent le même bien immobilier, l'incorporation de l'impôt sur les revenus locatifs dans l'impôt foncier, tout en réduisant leurs taux pourra être une réponse quant à ce. En outre, il faut solutionner les problèmes de mise en œuvre pour une bonne application.

En définitive, le système fiscal immobilier congolais doit être reformé par de législations físcales nouvelles, œuvrant à la rentabilité et à la simplicité, ainsi qu'à la justice sociale. Dans cette perspective, ces réformes ont pour principal objectif une amélioration de la structure du système fiscal congolais, pour un meilleur rendement de la collecte des impôts.

\section{BIBLIOGRAPHIE}

1. ARDANT G., Théorie sociologique de l'impôt, S.E.V.P.E.N, Paris, 1965

2. ARVIND ASHTA, Le fédéralisme fiscal : Australie, Canada, Inde, USA, les aspects fiscaux du droit budgétaire, ANRT, Paris, 2003.

3. BAKANDEJA WA MPUNGU, droit des finances publiques, éd. Noraf, Kinshasa, 1997

4. BAKANDEJA WA MPUNGU, les finances publiques : pour une meilleure gouvernance économique et financière en RDC, Larcier, Bruxelles, 2006

5. BELTRAME P. et MAHL L., techniques, politiques, et institutions fiscales comparées, 2éd. PUF, Paris, 1997.

6. BELTRAME P. et MEHL L., Techniques, politiques, et institutions fiscales comparées, 2éd. PUF, Paris, 1997.

7. BELTRAME P., les systèmes fiscaux, 3éd, PUF, paris, 1975.

47 BELTRAME P., les systèmes fiscaux, 3éd, PUF, paris, 1975, p. 70. 
8. BERNARD SALANIE, théorie économique de la fiscalité, Economica, Paris, 2002.

9. BIFUMANU NSOMPI, les réformes fiscales actuelles en RDC : la remise en question, PULL, Kinshasa, 2006.

10. BIRD R. et VAILLANCOURT F., Décentralisation financière et pays en développement : concepts, mesure et évaluation, in actualité économique, vol. 74, n 3, 1998.

11. Bocquet C., la traduction juridique : fondement et méthodologie, de Boeck, Bruxelles, 2008.

12. BOSEKOTA W'ATSHIA, Rebâtir le Congo Démocratique : De la Bonne Gouvernance Etatique et du Rôle Clé des PME-PMI!, éd. Presses Universitaires « BEL CAMPUS », Tome I, 2004.

13. CAMERON D., Property taxes, Willamette University, College of Law, 1999.

14. CHAMBAS G., BRUN JM., et GRAZIOSI G., La mobilisation de ressources propres locales en Afrique, l'atelier renforcement des capacités " gouvernances locales et Décentralisation, Vienne, 2007

15. Collet M., droit fiscal, 2ièmè éd., Thémis, Paris, 2009.

16. DECOSTER A. et VALENDUC C., l'impôt et la politique fiscale eFn Belgique, éd. Université de Bruxelles, Bruxelles, 2010.

17. DEFFAINS B. et FEREY S., « théorie du droit et analyse économique », Droits, $\mathrm{n}^{\circ} 45$, France, en ligne, 2007.

18. DELALANDE N et SPIRE A., Histoire sociale de l'impôt, La Découverte, Paris, 2010.

19. Duverger M, finances publiques, 8ième éd., PUF, Paris, 1975.

20. FOSTING JB., Le pouvoir fiscal en Afrique, LGDJ, Paris, 1995.

21. GAPYISI E., Le défi urbain en Afrique, Harmattan, Paris, 1989.

22. GEFFROY JB., grands problèmes fiscaux contemporains, Puf, paris, 1993.

23. GEFFROY JP., Grands problèmes fiscaux contemporains, Puf, paris, 1993.

24. GOTMAN B., l'enquête et ses méthodes : l'entretien, Nathan, tours, 1992.

25. GROSClAUDE J. et MARCHESSOU, Droit fiscal Général, 6e éd., Dalloz, Paris, 2007.

26. JOUMARD I., les systèmes fiscaux des pays de l'Union européenne, Revue économique de l'OCDE, 2002/1 no34.

27. KALINA KOLEVA ET MONNIER JM., la nature juridique de l'impôt dans l'ancienne et nouvelle économie du droit fiscal, Cahier de la maison des sciences économiques, Paris, 2006.

28. KIRAT T., " de l'analyse économique du droit à de nouvelles alliances? », in Revue économique, vol. 49, n4, Paris, 1998.

29. KIBANDA MATUNGILA et Cie, Le monstre appelé corruption, l'institut des stratégies pour le développement durable, Kinshasa, 2005

30. KITOPI KIMPINDE, des comportements économiques et manifestations de la résistance à l'impôt et de la nécessité des reformes fiscales et «économiques en RDC, thèse en Droit, Unilu, 2010. 
31. MACKAAY E. et POUSSEAU S., analyse économique du droit, édition Dalloz, Paris 2008.

32. MARSTBOOM L., Le cadastre et l'impôt, Wester, Bruxelles, 1946

33. MARTINEZ JC. et DI MALTA P., Droit fiscal contemporain : l'impôt, le fisc, le contribuable, T1, Litec, Paris, 1986

34. MIRRLEES J., « Les impôts, Pourquoi? Combien? », In: Revue française d'économie. Volume $15 \mathrm{~N}^{\circ} 4,2001 \mathrm{ANGE}$ BANGO, L'élaboration et la mise en œuvre de la fiscalité dans les pays de la communauté économique et monétaire de l'Afrique centrale (CEMAC), Thèse, Université Jean Moulin Lyon 3, 2009.

35. MPOY KADIMA, MPOY KADIMA, La rationalisation du système fiscal et douanier comme mécanisme relanceur de l'économie. Principes et outils d'une politique fiscale et douanière développementaliste, Editions Universitaires Africaines Kinshasa, Kinshasa, 2012

36. MUZANG KODI, Corruption et gouvernance en RDC durant la Transition (2003-2006), Institut D'études de Sécurité, Paris, 2008.

37. NGOASYVATHN P., Le rôle de l'impôt dans les pays en voie de développement, Tome1 LGDJ, 1974

38. NYABIRUNGU M. SONGA, La criminalisation de l'économie Zaïroise, éd. DES, Kinshasa, 1996.

39. OPPETIT B., Droit et économie, Archives de philosophie du droit, Paris, 1992

40. PAIN M., Kinshasa : la ville et la cité, ORSTOM, Paris, 1984

41. PIERRE BELTRAME et LUCIEN MEHL, techniques, politiques, et institutions fiscales comparées, 2éd. PUF, Paris, 1997

42. RAMBOURG M., " L'échec des réformes en Afrique. Le cas des pays francophones au sud du Sahara ", Revue Française d'Administration Publique, juillet-septembre 1998

43. RUBBERS, B., « Retour sur le «secteur informel». L'économie du Katanga (CongoZaïre) face à la falsification de la loi », Sociologie du travail, vol. 49, n³, Elsevier Masson SAS All, 2007

44. SALANIE B., théorie économique de la fiscalité, Economica, Paris, 2002.

45. SCHMIDT D, $l$ 'analyse économique du droit, Université de Panthéon, Paris 2, 2003.

46. SUSSET E., "Les enjeux de l'analyse économique du droit », in Labyrinthe, Actualité de la recherche $n^{\circ} 9$, Paris. 


\section{Impressum}

\section{KAS African Law Study Library - Librairie Africaine d'Etudes Juridiques}

Herausgeber: Konrad-Adenauer-Stiftung e.V., Klingelhöferstraße 23, 10785 Berlin

Schriftleitung: RA Prof. Dr. Hartmut Hamann, Falkertstraße 82, D-70193 Stuttgart Telefon: +49 (o) 711 120950-30 | Fax : +49 (o) 711 120950-50

Mail: hamann@hamann-legal.de

Erscheinungsweise: 4 Ausgaben pro Jahr

Druck und Verlag: Nomos Verlagsgesellschaft mbH \& Co. KG - Waldseestr. 3-5 D-76530 Baden-Baden. Telefon +49-7221-2104-0 / Fax 49-7221-2104-27

E-Mail:nomos@nomos.de

Anzeigen: sales friendly Verlagsdienstleistungen - Inh. Frau Bettina Roos - Pfaffenweg 15 - D-53227 Bonn Telefon +49-228-97898-o / Fax +49-228-97898-20

E-Mail: roos@sales-friendly.de

Urheber- und Verlagsrechte: Die Zeitschrift und alle in ihr enthaltenen einzelnen Beiträge sind urheberrechtlich geschützt. Jede Verwertung außerhalb der engen Grenzen des Urheberrechtsgesetzes ist ohne Zustimmung des Verlages unzulässig. Das gilt insbesondere für Vervielfältigungen, Übersetzungen, Mikroverfilmungen und die Einspeicherung und Verarbeitung in elektronischen Systemen. Der Nomos Verlag beachtet die Regeln des Börsenvereins des Deutschen Buchhandels e.V. zur Verwendung von Buchrezensionen.

ISSN 2363-6262

\section{Nomos}

